\title{
热裂法切割玻璃等硬脆材料关键技术研究进展
}

\author{
王海龙 ${ }^{1}$ ，王 扬 ${ }^{1}$ ，王向伟 ${ }^{2}$, 张宏志 ${ }^{1}$ \\ (1. 哈尔滨工业大学 机电工程学院, 哈尔滨 150001; 2. 哈尔滨商业大学, 哈尔滨 150028)
}

摘 要: 热裂法自 1967 年提出至今已取得丰硕成果。为使国内外学者清晰掌握其研究体系和发展趋势, 有必要对热 裂法近 60 年的研究成果进行规律性总结。本文首先明确提出热裂法的发展路线分两个方向: 非预制轨迹热裂切割 和预制轨迹热裂切割。在非预制轨迹热裂切割研究领域, 切割速度、裂纹轨迹偏移量和切割面质量是最主要的优化 目标参数; 在预制轨迹热裂切割研究领域, 切割速度和切割面质量是最受关注的优化目标参数。针对上述目标参数 的优化, 均可通过采用新的热源形式、冷却形式和优化工艺流程得以实现, 指出热裂法切割玻璃和陶瓷关键技术研 究方向在于创新发展新的热源技术、冷却技术和进一步优化工艺流程。

关 键 词: 热裂法; 玻璃; 陶瓷; 研究进展

中图分类号: TQ174 文献标识码: A

\section{Research Progress of Thermal Controlled Cracking of Hard-Brittle Plate}

\author{
WANG Hai-Long ${ }^{1}$, WANG Yang ${ }^{1}$, WANG Xiang-Wei ${ }^{2}$, ZHANG Hong-Zhi ${ }^{1}$
}

(1. School of Mechatronics Engineeing, Harbin Institute of Technology, Harbin 150001, China; 2. Harbin University of Commerce, Harbin 150028, China)

\begin{abstract}
Thermal Controlled Cracking Method (TCCM) has developed for more than 60 years with great success. The research progress of TCCM in the past 60 years was reviewed in two directions, i.e. Premade Trajectory Cutting (PTC) and Non-Premade Trajectory Cutting (NPTC) respectively. For PTC, cutting velocity, trajectory deviation and sectional quality are the main process parameters to be optimized. For NPTC, cutting velocity and sectional quality are the main process parameters to be optimized. The crucial technique for optimizing these above parameters is to adopt new techniques for generating advanced heating source, cooling source and better process flow. These new techniques are expected to provide enormous promotion for the developing of TCCM.
\end{abstract}

Key words: thermal controlled cracking; glass; ceramic; research progress

玻璃和陶瓷等硬脆材料在军事航天、电力电子、 汽车工业等领域均有广泛应用。如何高效高质量地 对上述材料进行切割加工一直是该领域亟需解决的 关键问题。切割加工方法主要有机械切割 ${ }^{[1-4]}$ 、激光 熔融切割 ${ }^{[5-6]}$ 、等离子切割 ${ }^{[7]}$ 、水射流切割 ${ }^{[8]}$ 、电火 花切割 ${ }^{[9-10]}$ 和水导激光切割 ${ }^{[11]}$ 等, 但上述方法均存 在切割质量差、切割效率低、噪音大等缺点, 无法 实现绿色环保等先进制造理念, 函需新的切割加工
方法。

热裂纹作为一种缺陷形式, 广泛存在于各种材 料之中, 是影响材料机械性能的关键因素之一[12-13]。 材料中裂纹的产生及扩展是材料科学与工程领域的

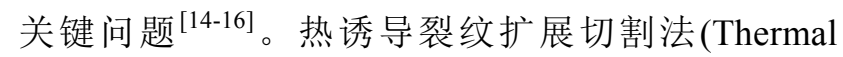
Cracking Method, 中文简称热裂法)是由美国学者 Lumley 等 1969 年提出并申请专利 ${ }^{[17]}$ 。该方法不再 将裂纹的产生与扩展视为影响材料性能的因素, 相

收稿日期: 2017-11-16; 收到修改稿日期：2018-01-07

基金项目: 国家自然科学基金(51275118) National Natural Science Foundation of China(51275118)

作者简介: 王海龙(1985-), 男, 博士研究生. E-mail: 13B908071@hit.edu.cn

通讯作者: 王向伟，教授. E-mail: 47817307@qq.com; 王 扬，教授. E-mail: wyyh@hit.edu.cn 
反, 该方法致力于发展诱导裂纹沿预定轨迹产生及 扩展的理论。该领域理论的发展将进一步丰富微宏 观材料断裂行为科学体系的内容, 并以此为基础开 发出适用于多种材料绿色环保的高效切割技术 ${ }^{[18-19]}$ 。

\section{1 热裂法应用现状}

热裂法基本原理是利用热源扫描产生的热应力 驱动并控制板材外表面初始裂纹沿预定轨迹扩展, 从而实现板材切割成型。

目前利用热裂法基本原理进行切割加工的成型 工艺有两种: 非预制轨迹热裂切割 ${ }^{[20]}$ 和预制轨迹热 裂切割 ${ }^{[21]}$, 分别见图 1 和图 2。非预制切割轨迹工 艺过程为: 首先在板材边缘处预制一定深度微裂纹, 然后用热源扫描产生的热应力驱动该裂纹沿扫描轨 迹扩展直至整个板材发生断裂, 最终实现切割。预 制切割轨迹工艺过程为: 首先在板材上表面或下表 面预制一定深度的切割轨迹, 然后用热源扫描产生 的热应力驱动切割轨迹向深度方向扩展直至整个板 材发生断裂。

热裂法属于非接触无材料损失加工, 切割过程 中无碎屑、无噪声, 具有切割质量好, 绿色环保等优 点, 在绿色制造领域独树一帜。经过近 60 年的发展, 该方法已发展为较有潜力的玻璃陶瓷等无机非金属 硬脆材料先进切割成型工艺, 能够满足一定市场需 求, 目前在裂片加工硅片、 LCD 液晶屏等领域得到 一定应用 ${ }^{[22-23]}$ 。

热裂法加工主要存在轨迹偏移、切割速度低和 表面质量不稳定等问题。本实验室通过总结国内外 学者多年研究成果, 发现开发多种热源形式、冷却 方式及优化工艺流程是解决上述问题的关键。下面 将按非预制轨迹热裂切割和预制轨迹热裂切割两种 工艺路线, 分别综述相关领域的研究历程和进展。

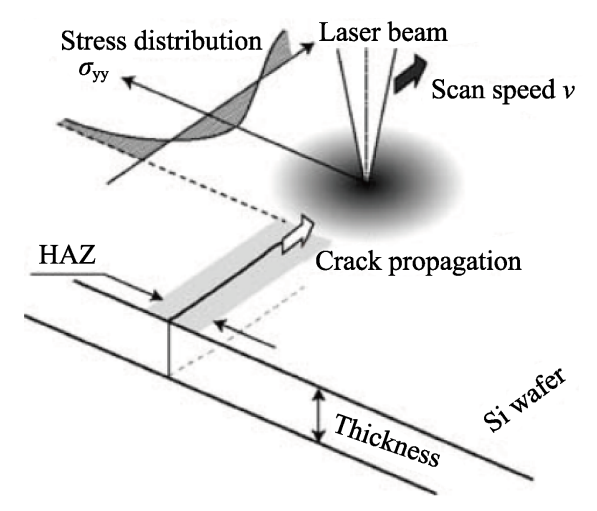

图 1 非预制轨迹热裂切割原理 ${ }^{[20]}$

Fig. 1 Mechanism of non-premade trajectory cutting(NPTC) ${ }^{[20]}$ (a)

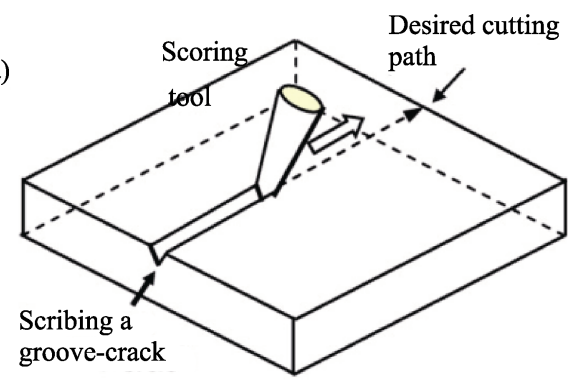

$\sqrt{2}$

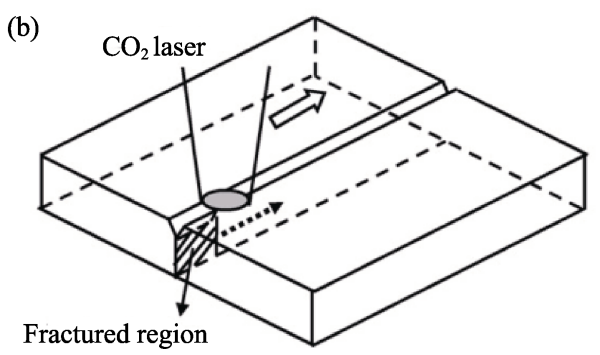

图 2 预制轨迹热裂切割原理 ${ }^{[21]}$

Fig. 2 Mechanism of premade trajectory cutting (PTC) ${ }^{[21]}$

\section{2 非预制轨迹热裂切割}

非预制轨迹热裂切割主要用于较厚玻璃和陶瓷 片的粗加工, 可以切割较厚板材, 在切割轨迹中间 部位没有碎屑和微裂纹, 切割表面质量较好。国内 外学者的研究目标是提升切割速度, 减小切割轨迹 与扫描轨迹偏移和改善切割表面质量。

\section{1 切割速度}

利用非预制轨迹热裂法切割玻璃片时，提升切 割速度的一种有效方法是改变热源能量分布形状和 施加冷却。Yamamoto 等 ${ }^{[24-29]}$ 采用椭圆形分布 $\mathrm{CO}_{2}$ 激光+水冷的方法对玻璃进行热裂切割, 首先用金 刚石砂轮在划切路径首端一侧玻璃平板边缘上表面 预制裂纹缺口, 然后用椭圆形 $\mathrm{CO}_{2}$ 激光光斑进行热 裂, 激光光斑尺寸为 $22 \mathrm{~mm} \times 2.1 \mathrm{~mm}$, 最后在其扫描 方向光斑后方随动喷水进行冷却以完成热裂切割。 研究表明, 提高激光热源功率或施加冷却措施可使 初始裂纹处的应力强度因子快速达到阈值, 以此提 升切割速度。2010 年美国康宁公司 Abramov 等 ${ }^{[30-31]}$ 采用激光诱导热裂切割化学强化玻璃,他们采用线 状光斑 (随圆光斑长轴长度与短轴长度的比值极大) 的 $\mathrm{CO}_{2}$ 激光, 对中心拉应力为 $26 \sim 45 \mathrm{MPa}$ 的 $1 \mathrm{~mm}$ 厚的强化玻璃进行了激光诱导热裂切割。2011 年, 他们又实现了 $1000 \mathrm{~mm} / \mathrm{s}$ 的玻璃切割速度, 并且将切 割后材料边缘处的残余应力值控制在 $3.49 \mathrm{MPa}$ 以下。 提高切割速度的另一个有效方法是采用体加热 
热源。日本 LEMI 公司基于此研发了多款激光诱导 热裂切割机, 提出了当激光波长处于 $\mathrm{CO}_{2}$ 激光 $(10.6 \mu \mathrm{m})$ 和 $Y A G$ 激光 $(1.06 \mu \mathrm{m})$ 之间时, 激光能够透过玻璃表 面射入玻璃内部，并在内部全部吸收而不射出玻璃 (即体加热热源)，此时能够达到较高速度的热裂法 切割。该公司的研究人员 ${ }^{[32]}$ 给出了提高切割速度的 多种解决方案：用线光源(粗圆型光斑)替代常用的 圆形光斑，通过提高热源面积可以提高切割速度 3 倍; 用表面热源进行加工，在材料上方 $200 \mathrm{~mm}$ 处布置 输出功率为 $1 \mathrm{~kW}$ 的管形红外灯 (实现体加热), 叠加 在 LD 激光扫描上，扫过 $580 \mathrm{~mm}$ 宽的玻璃中心线, 可以获得 $300 \mathrm{~mm} / \mathrm{s}$ 的切割速度, 而不加红外灯的切 割速度仅 $23 \mathrm{~mm} / \mathrm{s}$, 提高了 13 倍 ${ }^{[33]}$ 。该文献还给出了 切割速度随平板宽度变化的曲线, 其中宽度为 $2000 \mathrm{~mm}$ 的玻璃板的切割速度为 $10 \mathrm{~mm} / \mathrm{s}$, 见图 $3^{[33]}$ 。

\section{2 裂纹轨迹偏移}

裂纹轨迹偏移一直是非预制轨迹热裂法亟需解 决的问题。2009 年, Salman 等 ${ }^{[34]}$ 对二极管激光热裂 法切割玻璃进行了一系列研究, 他们采用功率为 $770 \mathrm{~W}$ 、波长为 $808 \sim 940 \mathrm{~nm}$ 、光斑直径为 $3 \mathrm{~mm}$ 的 二极管连续式激光, 以 $33 \mathrm{~mm} / \mathrm{s}$ 的速度切割 $5 \mathrm{~mm}$ 厚 的钠钙玻璃, 指出在材料的切入口和切出口处存在 严重的轨迹偏移现象, 偏移角度在 $10^{\circ}$ 左右, 见图 4。 他们对切割过程中的工件应力场进行了仿真分析, 研究发现切入口和切出口处的拉应力过大，使得 轨迹发生偏移。同年, Salman 等 ${ }^{[35]}$ 在完成连续式激 光对称切割玻璃的研究后, 又对脉冲激光热裂法对 称切割玻璃进行了研究, 指出脉冲参数和切割速度 对于切入口和切出口处的偏移角度和切割表面质量 的影响关系, 结果表明采用脉冲激光可以减少能 量的输入, 降低温度变化, 热应力也相应减小, 从 而有效地减小切入口和切出口处的轨迹偏移值。

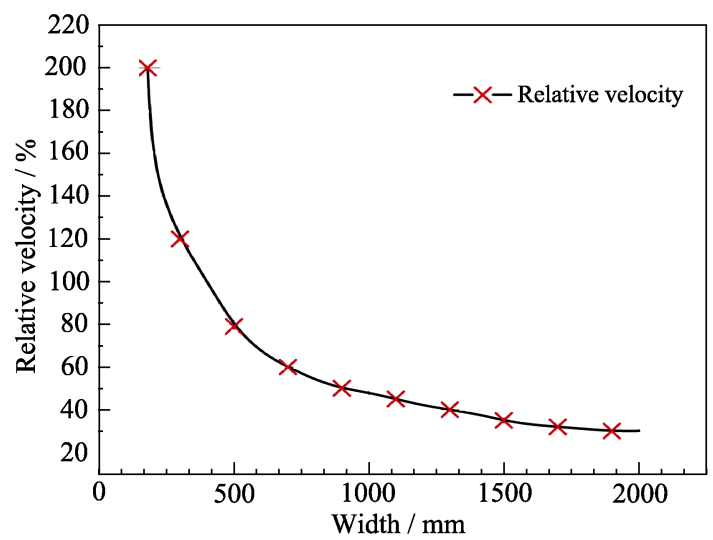

图 3 玻璃平板宽度对切割速度的影响规律曲线 ${ }^{[33]}$

Fig. 3 Cutting velocity-plate width curve ${ }^{[33]}$
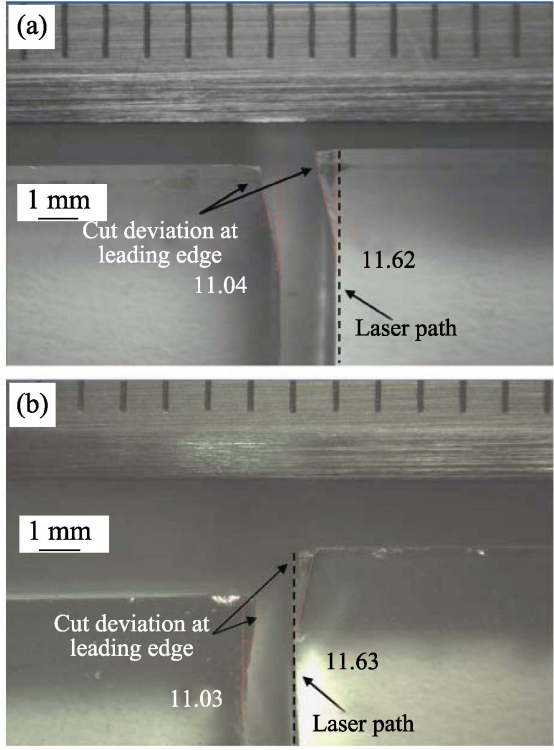

图 4 非预制轨迹热裂切割出入口偏移现象 ${ }^{[34]}$

Fig. 4 Trajectory deviation of NPTC ${ }^{[34]}$

采用占空比为 0.6 的脉冲激光可以减小轨迹角度偏 移量和轨迹位移偏移量的 $36 \%$ (与连续式激光所产 生的轨迹偏移相比)。2010 年, Salman 等 ${ }^{[36]}$ 为了得到 切入口、切出口处最小的轨迹偏移量, 就不同的激 光光斑形状对切割轨迹偏移的影响进行了研究, 比 较了圆形、长方形短边、长方形长边、正三角形、 反三角形和菱形光斑的温度场和应力场, 并通过仿 真与实验结果的对比表明: 正三角形光斑在切入口 处使得拉应力变化较小, 可以起到改善轨迹偏移的 作用;而反三角形光斑在切出口处也有相同的作用。 另外，他们还指出激光的发散角对于改善切入口、 切出口处的轨迹偏移有着重要作用。

2015 年, Salman 等 ${ }^{[37]}$ 还针对切入口和切出口处 的应力分布状态从仿真角度进行了深入研究, 分别 应用人工神经网络和有限元模型，预测不同玻璃板 厚度和激光扫描速度时切入口和切出口处的热应力, 并比较两种模型的效果。结果表明, 人工神经网络模型 的预测结果要比有限元模型的预测结果更好。

日本长冈科学技术大学 Miyashita 等 ${ }^{[38]}$ 在裂纹 尖端的前后方各布置一束激光, 形成姊妹激光光束 (见图 5), 并通过移动裂纹尖端后方光束使得两束 激光连线沿着裂纹扩展偏转的方向,通过调节强度 比例来控制裂纹扩展的方向。研究表明二者的强度 比例可以显著影响到 II 型裂纹强度因子。Miyashita 等利用该方法实现了 30 度裂纹扩展偏转的切割,

哈尔滨工业大学杨立军等 ${ }^{[39]}$ 应用 YAG 激光对 玻璃实现了体加热热裂法切割, 研究发现 YAG 激 光可以穿透玻璃, 进行体吸收形式的切割, 其原理 
见图 6。在板类和管类玻璃件上切割曲线轨迹有很 大的优越性，见图 7 。

\section{3 玻璃切割面强度}

玻璃切割面的强度与切割方法直接相关。热裂 法切割玻璃获得的表面质量有效地提升了玻璃板的 弯曲强度。2003 年德国 $\mathrm{Silvio}^{[40]}$ 总结了用激光加工 脆性材料的方法, 并对各种方法切割 $3 \mathrm{~mm}$ 厚玻璃 所得到的加工边缘强度进行了对比, 如图 8 所示。

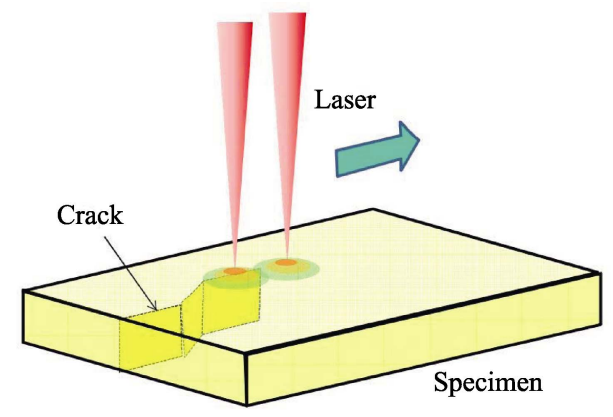

(a)

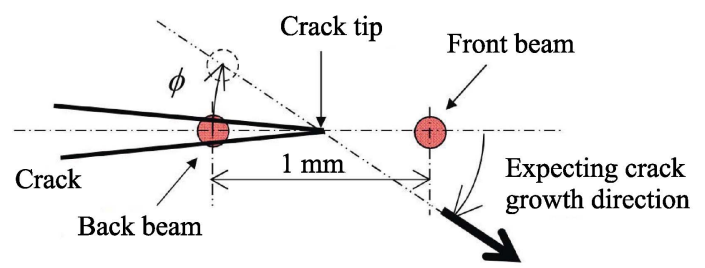

(b)

图 5 双束激光完成轨迹偏转切割原理 ${ }^{[38]}$

Fig. 5 Double laser beam for cutting with turn ${ }^{[38]}$

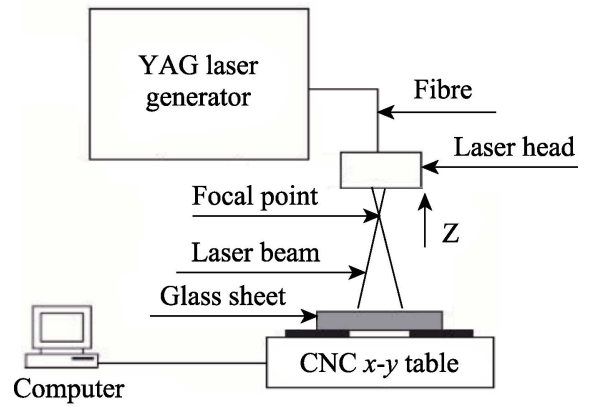

图 6 YGA 激光体加热热裂切割原理图 ${ }^{[39]}$

Fig. 6 YAG laser cutting glass based on volumetric heating method $^{[39]}$
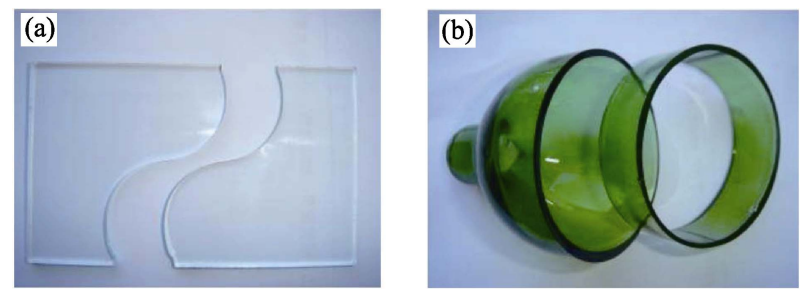

图 7 YGA 体加热激光热裂切割玻璃样品 ${ }^{[39]}$

Fig. 7 Glass cut by NPTC with YAG ${ }^{[39]}$

(a) Plate; (b) Tube

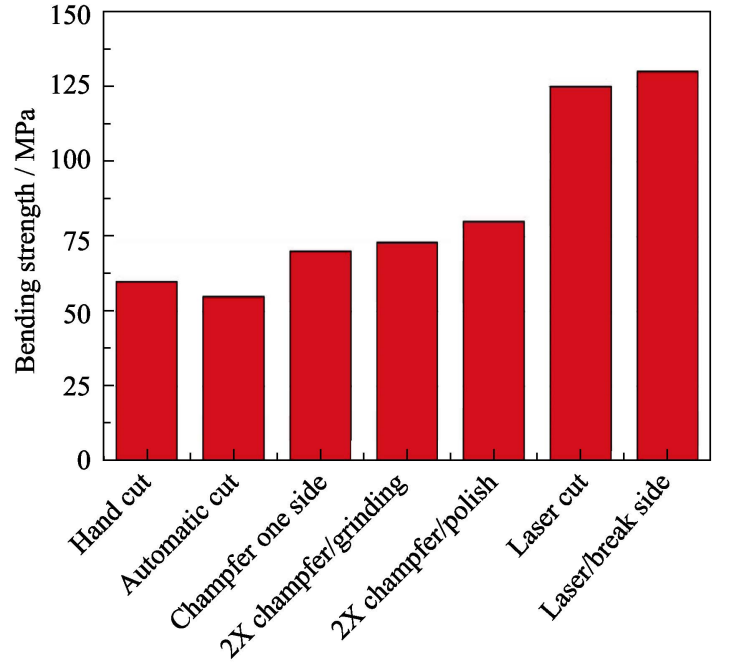

图 8 不同加工方法的加工边缘强度对比 ${ }^{[40]}$

Fig. 8 Comparison of bending strength of cutting section with multiple cut methods ${ }^{[40]}$

2009 年富士康公司与俄罗斯莫斯科国立仪器 与信息大学 Kondratenko 等 ${ }^{[41]}$ 研究了激光诱导热裂 切割厚度达 4 19 $\mathrm{mm}$ 的玻璃, 他们采用 $\mathrm{CO}_{2}$ 激光(实 际用 20 100 W), 双透镜(柱镜+球镜)形成线形光斑 (长约 40 60 mm, 宽约 1.5 2.5 mm), 辅以空气一水混 合冷却法控制工艺过程中的开裂阶段，对 $800 \mathrm{~mm} \times$ $800 \mathrm{~mm} \times 4 \mathrm{~mm}$ 的玻璃进行了切割, 并对用机械、磨 削及激光切割的 $6 \mathrm{~mm}$ 厚玻璃的强度进行了比较, 得到激光诱导热裂切割玻璃由于断面无表面微裂纹, 质量较好, 其边缘强度是传统机械切割强度的 5.5 倍。

断面质量是影响板材强度的关键因素。对于双 层玻璃, 提升断面质量的有效方法是使上下层同时 得到加热和冷却。2014 年, 韩国 Choi 等 ${ }^{[42]}$ 提出在 第一层玻璃下表面涂炭, 并通过脉冲 YAG 激光扫 描该玻璃产生等离子体, 该等离子体作用在第二层 玻璃上, 改变该层玻璃的光学特性, 同时产生局部 的快速加热和冷却, 使得第二层玻璃开裂分离, 其 原理如图 9 所示。研究指出激光参数(激光能量与脉 冲宽度)和两层玻璃间的距离是影响切割质量的主 要因素, 给出了一组最优参数, 即当激光峰值功率密 度为 $45 \sim 50 \mathrm{GW} / \mathrm{cm}^{2}$, 玻璃间距为 $150 \mu \mathrm{m}$, 脉冲宽 度为 $4 \mathrm{~ns}$ 时, 断面的质量最好。

除了激光热源外, 2016 年 Wang 等 ${ }^{[43]}$ 利用短边 收缩聚焦波导, 成功地将微波聚焦成粗圆线型体加 热光斑, 实现了同时体加热切割较厚玻璃和碳化硅 陶瓷片的突破性进展, 其原理和切割结果分别见图 10 和图 11。研究指出, 短边收缩聚焦波导产生的热 源为粗圆形体加热微波热源, 热源在厚度方向透过 性较激光热源好, 能够对厚度方向的非透明和透明 材料进行较均匀的加热。由此可知, 利用较低的 


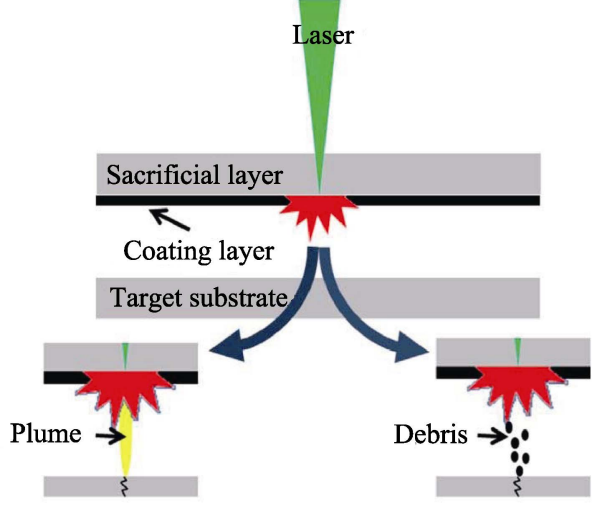

图 9 激光诱导等离子体加工玻璃原理图 ${ }^{[42]}$

Fig. 9 Principle diagram of laser induced plasma processing glass $^{[42]}$

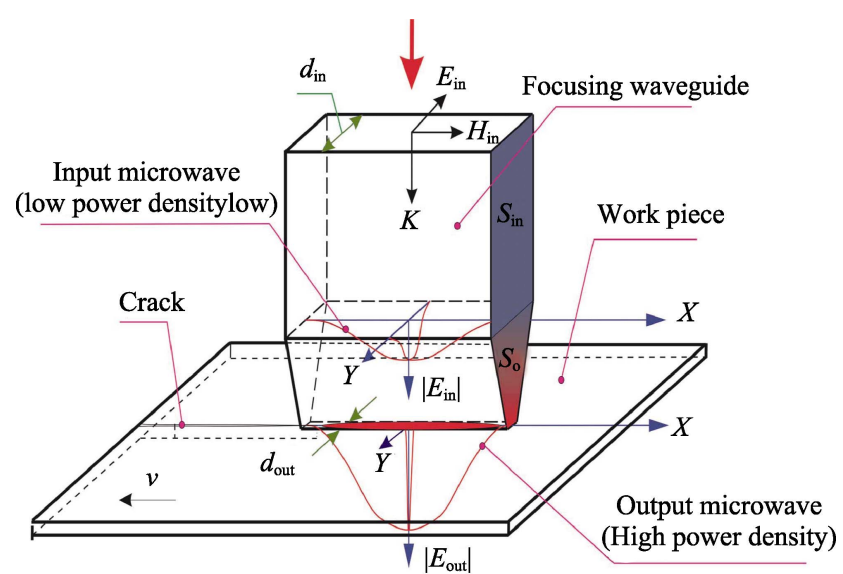

图 10 微波热裂法切割实验装置图 ${ }^{[43]}$

Fig. 10 Equipment for microwave induced cracking(MIC) ${ }^{[43]}$

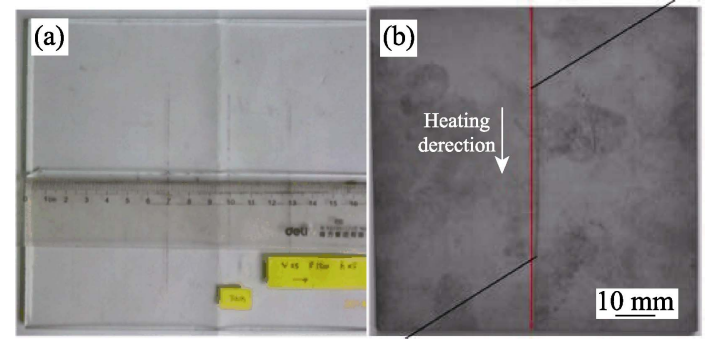

图 11 微波热裂法切割玻璃及(a) SiC 陶瓷片(b)的结果 ${ }^{[43]}$

Fig. 11 (a) Glass and (b) $\mathrm{SiC}$ ceramic plates cut with $\mathrm{MIC}^{[43]}$

温度即可产生驱动初始裂纹扩展的热应力。另外, 与其他激光热源相比，椭圆形微波热源在加热区域 将产生较长的内部压应力区，该压应力区将使裂纹扩 展过程更稳定、可控，切割断面质量较好。

\section{3 预制轨迹热裂切割}

预制裂纹扩展切割又称划槽热裂切割, 主要用 于液晶显示(LCD)、等离子显示(PDP)和平板显示 (FPD)等玻璃的裂片加工, 特点是切割速度较快和
切割轨迹精度较高。由于预制轨迹热裂切割没有轨 迹偏移的现象，因此国内外学者关注点在于如何提 升切割速度、提升切割表面质量和拓展该方法的适 用板材类型。

\section{1 切割速度}

2002 年, Kang 等 ${ }^{[44]}$ 对夹层玻璃进行高速切割时, 变换了激光光束的形状(采用更长的椭圆形光斑)和 冷却介质(采用液体冷却), 实现了夹层玻璃的高速 切割。对于 PDP 的切割, 作者采用先划切再热裂两 步切割的方法, 其效率要优于整体一次切割的方法, 并基于此原理研发出系列加工装置。

2009 年, Tsai 等 ${ }^{[21]}$ 采用金刚石刻划加脉冲式 $\mathrm{CO}_{2}$ 激光热裂法切割玻璃, 他们提出了在切割轨迹 中部利用激光热裂法促进裂纹扩展的两种方法, 并 结合槽底裂纹扩展的方式，提出了一种双方向切割 玻璃芯片的策略，提高了切割效率。

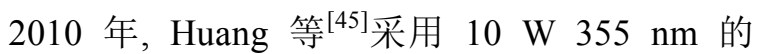
$\mathrm{Nd}$ : YAG 激光器在玻璃内部产生隐性裂纹后, 通过 $40 \mathrm{~W}$ 的 $\mathrm{CO}_{2}$ 激光器使得裂纹扩展, 完成玻璃基底的 切割, 实验原理及结果见图 12。2015 年, Huang 等 ${ }^{[46]}$ 又将超声振动引入到 $\mathrm{Nd}$ : $\mathrm{YAG}$ 紫外激光与连续式 $\mathrm{CO}_{2}$ 激光的切割系统中, 进行了 $\mathrm{LCD}$ 玻璃基板的切 割研究, 结果表明超声振动的引入可以很好地提高 切割速度(原有速度的三倍)。

2012 年, $\mathrm{Su}$ 等 ${ }^{[47]}$ 将生产触摸屏板切割工艺流程 分为以下四步：抓取玻璃母板，金刚石轮预制边缘 裂纹(几个微米深度), $\mathrm{CO}_{2}$ 激光+冷却进行刻划, 双 向交叉轨迹刻划。工艺过程如图 13 所示, 首先划第 一方向, 全部划切成条状后再转至第二方向, 采用 相同工艺进行划切; 后划先羒法 LSFB(Last-ScribingFirst-Breaking), 即先沿着第二方向秎开, 羒成条状 后再将其沿着第一方向的划切裂缝扮开; 成品检 测。Su等针对该工艺流程通过对重要参数进行识别; 实验与数据采集, 模型结构预测, 以及参数优化等 手段, 不仅提高了切割效率, 更获得了较好的切割 质量。

\section{2 切割表面质量}

2008 年, $\mathrm{KIM}$ 等 ${ }^{[48]}$ 采用飞秒激光刻蚀代替机械 方法划槽, 并用 $\mathrm{CO}_{2}$ 激光热裂替代真空吸盘分离 $\mathrm{LCD}$ 玻璃。这种方法减小了玻璃在划刻阶段的损伤, 通过优化飞秒激光的参数, 使得所划槽的边缘没有 微裂纹。实验结果表明在飞秒激光脉冲个数较少时, 由于玻璃的损伤太小, 划槽深度不够, 切割质量较 差; 而当飞秒激光脉冲个数增加至六个脉冲之后, 跟随的 $\mathrm{CO}_{2}$ 激光通过热应力分离玻璃的效果较好, 见图 14。 

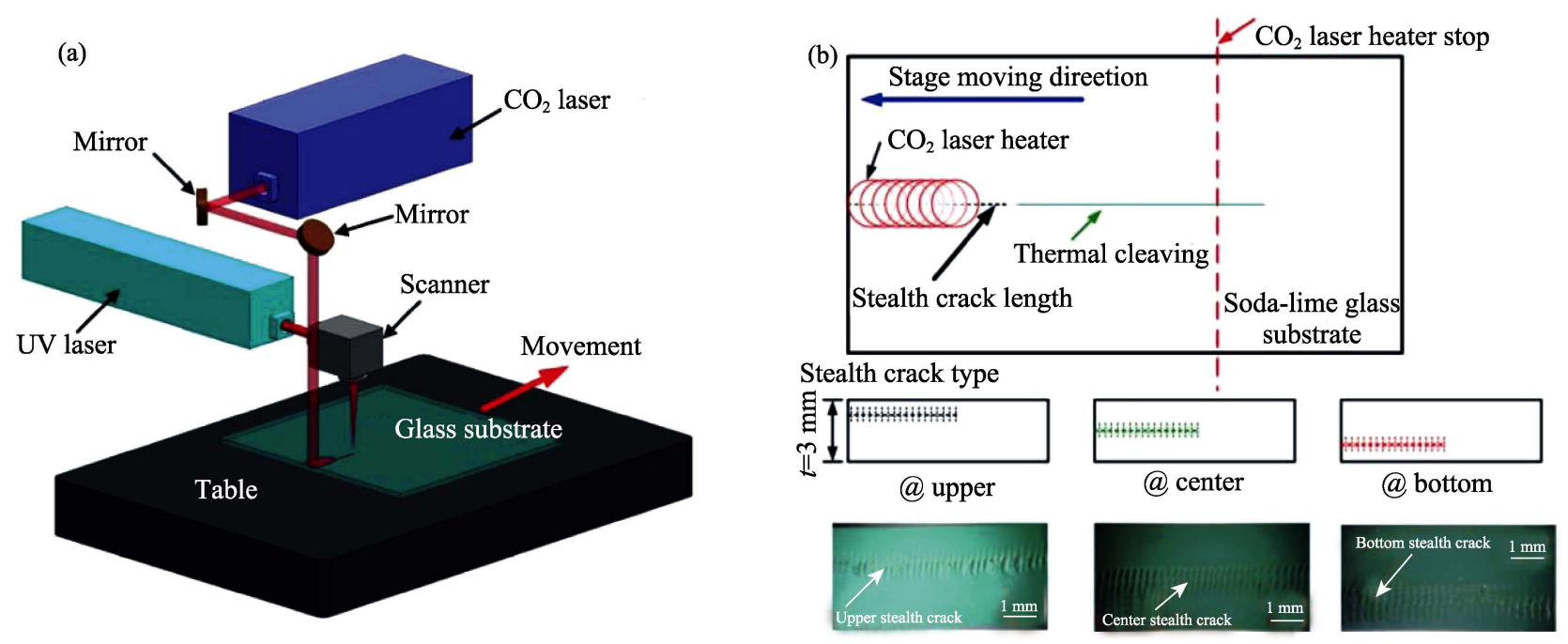

图 12 隐纹产生原理及布置位置 ${ }^{[45]}$

Fig. 12 Inner initial fracture induced method and its location ${ }^{[45]}$

(a) Method; (b) Location

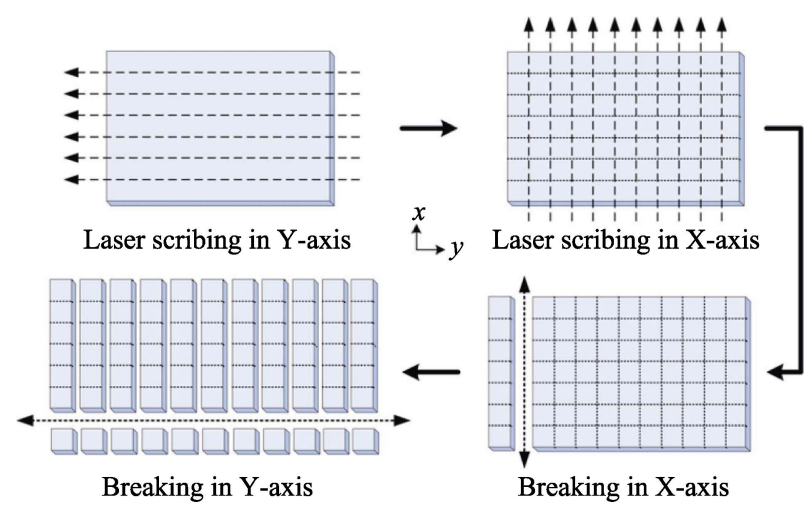

图 13 预制轨迹切割法切割液晶玻璃板工艺流程 ${ }^{[47]}$

Fig. 13 Process flow of splitting with PTC $\mathrm{P}^{[47]}$
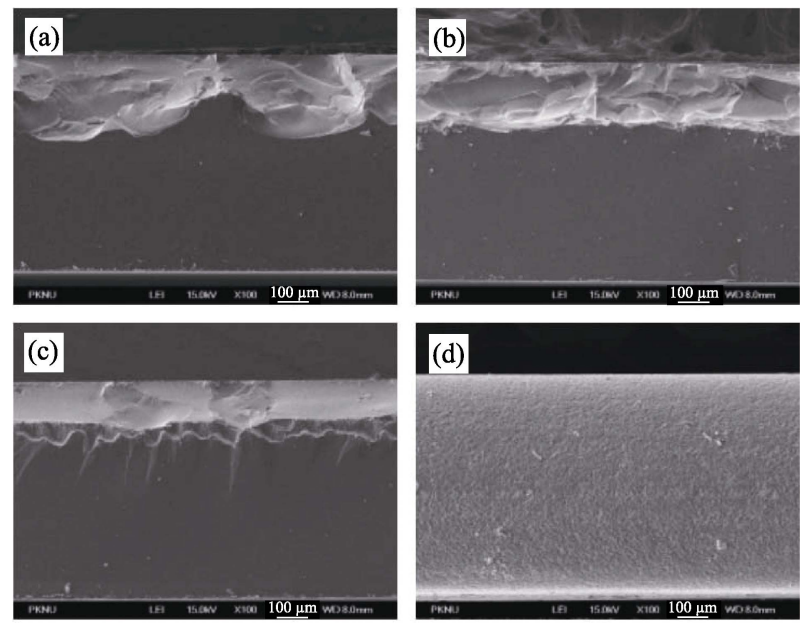

图 14 飞秒划切 $\mathrm{CO}_{2}$ 激光热裂切割玻璃截面照片 ${ }^{[48]}$

Fig. 14 Cross-sectional views of femtosecond laser scribing followed by $\mathrm{CO}_{2}$ laser heating and rapid cooling ${ }^{[48]}$

(a) After one laser pass; (b) After five laser passes; (c) After six laser passes; (d) Initial crack propagation

2008 年, Tsai 等 ${ }^{[21,49]}$ 利用金刚石刀具刻划并用
连续式 $\mathrm{CO}_{2}$ 激光沿划槽扫描, 使得滑槽裂纹在整个 玻璃的厚度上扩展的方法切割了 $0.63 \mathrm{~mm}$ 厚的 LCD 玻璃基板。由于金刚石刀具刻划时，槽底裂纹的大 小和方向的不确定性, 使得加工后的分离表面不垂 直于平板玻璃的表面。

2011 年, 汪旭煌等 ${ }^{[50-51]}$ 针对液晶玻璃基板进行 了激光热裂法切割研究, 他们提出了一种新的划槽 热裂切割加工方法, 即先用 YAG 激光的瞬间高能 量使液晶玻璃表面产生微裂纹, 然后用 $\mathrm{CO}_{2}$ 激光作 为热源对其进行加热, 并通过 $\mathrm{Ar}$ 气对其进行冷 却, 分析了激光参数与玻璃基板厚度对切割质量的 影响。

\section{4 总结与展望}

热诱导裂纹扩展切割法利用毛坏原有裂纹缺陷 的诱导扩展进行切割加工, 具有绿色、环保、高效 等优点, 已引起国内外学者广泛而深入的研究。热 诱导裂纹扩展切割法又分为非预制轨迹热裂切割和 预制轨迹热裂切割。

1) 非预制轨迹热裂切割的优化目标参数为切 割速度、轨迹偏移和断面质量。通过优化热源类型 及其能量分布特性和施加冷却等辅助装置, 可以有 效提高非预制轨迹热裂切割的切割速度, 减小轨迹 偏移，并获得较好的断面质量。

2) 相比较而言，预制轨迹热裂切割并没有轨 迹偏移的现象, 其优化目标参数为切割速度和切割 断面质量。非预制轨迹热裂切割可采取弯曲应力、 超声振动和冷却等措施与热裂法复合, 并采取一定 的切割顺序策略, 可得到较好的切割质量, 较快的 
切割速度。

3) 无论非预制轨迹还是预制轨迹热裂切割, 开发多种热源形式(微波和激光)、冷却形式、应力 施加方法、超声振动复合方法等关键技术, 都能够 拓展该加工方法的适用材料范围(玻璃、硅片及陶 瓷)，获得较快的切割速度、较好的加工质量，是热 裂法切割加工的发展趋势。随着该方法的不断完善, 必将在绿色加工领域发挥更大的作用。

\section{参考文献:}

[1] ZHANG CHONG, YUAN SONG-MEI, AMIN MUHAMMAD, et al. Development of a cutting force prediction model based on brittle fracture for $\mathrm{C} / \mathrm{SiC}$ in rotary ultrasonic facing milling. International Journal of Advanced Manufacturing Technology, 2016, 85(1-4): 573-583.

[2] SENER KARABULUT, HENIFI CINICI, HALIL KARAKOC. Experimental investigation and optimization of cutting force and tool wear in milling Al7075 and open-cell SiC foam composite. Arabian Journal for Science \& Engineering, 2016, 41(5): 17971812.

[3] ZHAO GUO-LONG, HUANG CHUANZHEN, HE NING, et al. Fabrication and cutting performance of reactively hot-pressed $\mathrm{TiB}_{2}$-TiC-SiC ternary cutting tool in hard turning of AISI H13 steel. International Journal of Advanced Manufacturing Technology, 2017, 91: 943-954.

[4] YUAN SONG-MEI, FAN HUITAO, AMIN MUHANMMAD, et al. A cutting force prediction dynamic model for side milling of ceramic matrix composites $\mathrm{C} / \mathrm{SiC}$ based on rotary ultrasonic machining. International Journal of Advanced Manufacturing Technology, 2016, 86(1-4): 37-48.

[5] SHARMA VIKAS, VINOD KUMAR. Investigating the quality characteristics of $\mathrm{Al} 5052 / \mathrm{SiC}$ metal matrix composites machined by $\mathrm{CO}_{2}$ laser curve cutting. Proceedings of the Institution of Mechanical Engineers Part L: Journal of Materials Design \& Applications, 2015, 232(1): 3-19.

[6] SHIN DONGSIG, YONGKWON CHO, HYONKEE SOHN. Towards optimized efficiency of ablation in copper using a $515 \mathrm{~nm}$ picosecond laser process. Journal of Laser Micro, 2015, 10(3): 241-244.

[7] YASUHISA SANO, KOHEI AIDA, TAKEHIRO KATO, et al. Cutting of SiC wafer by atmospheric-pressure plasma etching with wire electrode. Materials Science Forum, 2012, 717-720: 865-868.

[8] SAVRUN E, TAYA M. Surface characterization of SiC whisker/2124 aluminium and $\mathrm{Al}_{2} \mathrm{O}_{3}$, composites machined by abrasive water jet. Journal of Materials Science, 1988, 23(4): 1453-1458.

[9] SON HYEON-TAEK, KIM DAE-GUEN, PARK SOON-SUB, et al. Spark plasma sintering and ultra-precision machining characteristics of SiC. Korean Journal of Materials Research, 2010, 20(11): 559-569.

[10] PRASHANTHA S, VEERESHA R B, SHASHIDHARA S M, et al. A study on machining characteristics of Al6061-SiC metal matrix composite through wire-cut electro discharge machining. Materials Today Proceedings, 2017, 4(10): 10779-10785.

[11] ZHOU WEIGUO, GONG KEYU, WAN JIE, et al. Molecular dynamics simulation study on ablation of silicon by water-jet-guided laser. Proceedings of the Institution of Mechanical Engineers Part E Journal of Process Mechanical Engineering, 2017, 231(6): $1217-1225$.

[12] DANIEL LAROUCHE, JOSEPH LANGLAIS, WU WEI-LI, et al. A constitutive model for the tensile deformation of a binary aluminum alloy at high fractions of solid. Metallurgical \& Materials Transactions B, 2006, 37(3): 431-443.

[13] SUYITNO, KOOL W H, KATGERMAN L. Integrated approach for prediction of hot tearing. Metallurgical \& Materials Transactions A, 2009, 40(10): 2388-2400.

[14] RACHID MHAMDIA, SERIER B, AIBEDAH A, et al. Numerical analysis of the influences of thermal stresses on the efficiency of bonded composite repair of cracked metallic panels. Journal of Composite Materials, 2017, 51(26): 3701-3709.

[15] BILČÍK J, SONNENSCHEIN R, GAŽOVIČOVÁ N. Causes of early-age thermal cracking of concrete foundation slabs and their reinforcement to control the cracking. Slovak Journal of Civil Engineering, 2017, 25(3): 8-14.

[16] CHU DONG-YANG, LI XIANG, LIU ZHANLI. Study the dynamic crack path in brittle material under thermal shock loading by phase field modeling. International Journal of Fracture, 2017, 330: $1-16$.

[17] GRAHAM C E, LUMLEY R M, OBERHOLZER D J. Laser Substrate Parting: US, US3629545. 1971.

[18] KARLSSON M N A, DEPPERT K, WACASER B A, et al. Size-controlled nanoparticles by thermal cracking of iron pentacarbonyl. Applied Physics A, 2005, 80(7): 1579-1583.

[19] FRIEDL D I. Method for Laser Thermal Separation of Ceramic or Other Brittle Flat Materials: EP, EP1990168. 2012.06.27.

[20] UEDA T, YAMADA K, OISO K, et al. Thermal stress cleaving of brittle materials by laser beam. CIRP Annals - Manufacturing Technology, 2002, 51(1): 149-152.

[21] TSAI CHWAN-HUEI, HUANG BO-WEN. Diamond scribing and laser breaking for LCD glass substrates. Journal of Materials Processing Technology, 2008, 198(1/2/3): 350-358.

[22] SHEPELOV G V, SHIGANOV I N. Cutting sheet glass with the beam of a solid-state laser. Welding International, 2000, 14(12): 988-991.

[23] KONDRATENKO VLADIMIR S. Method of Splitting Non-metallic Materials: US, US 5609284 A. 1997.

[24] YAMAMOTO KOJI, HASAKA NOBORU, MORITA HIDEKI, et al. Thermal stress analysis on laser scribing of glass. Journal of Laser Applications, 2008, 20(4): 193-200.

[25] YAMAMOTO KOJI, HASAKA NOBORU, MORITA HIDEKI, et al. Three-dimensional thermal stress analysis on laser scribing of glass. Precision Engineering, 2008, 32(4): 301-308.

[26] YAMAMOTO KOJI, HASAKA NOBORU, MORITA HIDEKI, et al. Partial growth of crack in laser scribing of glass. Journal of Laser Applications, 2009, 21(2): 917-923.

[27] YAMAMOTO KOJI, HASAKA NOBORU, MORITA HIDEKI, et al. Influence of thermal expansion coefficient in laser scribing of glass. Precision Engineering, 2010, 34(1): 70-75.

[28] YAMAMOTO KOJI, HASAKA NOBORU, MORITA HIDEKI, et al. Influence of glass substrate thickness in laser scribing of glass. Precision Engineering, 2010, 34(1): 55-61.

[29] YAHATA KEISUKE, YAMAMOTO KOJI, ETSUJI OHMURA. Crack propagation analysis in laser scribing of glass (machine elements, design and manufacturing). Journal of Laser Micro, 2010, 5(2): 109-114. 
[30] ABRAMOV ANATOLI A, Black MATTHEW L, GLAESEMANN G SCOTT. Laser separation of chemically strengthened glass. Physics Procedia, 2010, 5: 285-290.

[31] ABRAMOV ANATOLI A, SUN YA-WEI, XU WEI, et al. Laser Scoring of Glass Sheets at High Speeds and With Low Residual Stress: US, US8011207. 2011.

[32] NORIO KARUBE, HIROSHI MIURA. Full-body Laser Scribing Method of Fragile Material: US, US20070062921. 2007.

[33] KOJIRO KARUBE, NORIO KARUBE. Laser-induced cleavage of LCD glass as full-body cutting. Proceedings of SPIE, 2008, 6880: $1-10$.

[34] SALMAN NISAR, SHEIKH M A, LI LIN, et al. Effect of thermal stresses on chip-free diode laser cutting of glass. Optics \& Laser Technology, 2009, 41(3): 318-327.

[35] SALMAN NISAR, KHAN SOHAIB Z, ALI MUHAMMAD, et al. Comparison of cut path deviation between $\mathrm{CO}_{2}$, and diode lasers in float-glass cutting. Journal of Russian Laser Research, 2014, 35(2): 182-192.

[36] SALMAN NISAR, SHEIKH M A, LI LIN, et al. The effect of laser beam geometry on cut path deviation in diode laser chip-free cutting of glass. Journal of Manufacturing Science \& Engineering, 2010, 132(1): 165-174.

[37] SALMAN NISAR, LI LIN, ALI MUHAMMAD, et al. The effect of continuous and pulsed beam modes on cut path deviation in diode laser cutting of glass. International Journal of Advanced Manufacturing Technology, 2010, 49(1-4): 167-175.

[38] MIYASHITA YUKIO, MASASHI MOGI, HIROTAKA HASEGAWA, et al. Study on a controlling method for crack nucleation and propagation behavior in laser cutting of glass. Journal of Solid Mechanics and Materials Engineering, 2008, 2(12): 15551566.

[39] CAI NA, YANG LI-JUN, WANG YANG, et al. Experimental research of YAG laser cutting soda-lime glass sheets with controlled fracture. Key Engineering Materials, 2010, 431-432: 507-510.

[40] SILVIO GEORGI. Separating and structuring of brittle material by the use of laser radiation. Proceedings of SPIE, 2003, 5063: 426-431.

[41] SHALUPAEV S V, SHERSHNEV E B, NIKITYUK YU V, et al.
Two-beam laser thermal cleavage of brittle nonmetallic materials. Journal of Optics Technology, 2006, 73(5): 356-359.

[42] CHOI WON-SEOK, KIM JONG-HYEONG, KIM JOOHAN, Thermal cleavage on glass by a laser-induced plume. Optics \& Lasers in Engineering, 2014, 53(2): 60-68.

[43] WANG HAI-LONG, ZHANG HONG-ZHI, WANG-YANG. Splitting of glass and $\mathrm{SiC}$ ceramic sheets using controlled fracture technique with elliptic microwave spot. Ceramics International, 2017, 43(2): 1669-1676.

[44] HANG KYOUNG-SHIK, HONG SOON-KUG,OH SEOK-CHANG, et al. A study of cutting glass by laser. Proceedings of SPIE, 2002, 4426: $367-370$.

[45] HUANG KUO-CHENG, WU WEN-HONG, TSENG SHIH-FENG, et al. The mixed processing models development of thermal fracture and laser ablation on glass substrate. International Conference on Advances in Materials and Processing Technologies, 2010, 1: 1612-1617.

[46] HUANG KUO-CHENG, HSIAO WEN-TSE, HWANG CHI-HUNG, et al. The laser ablation model development of glass substrate cutting assisted with the thermal fracture and ultrasonic mechanisms. Optics \& Lasers in Engineering, 2015, 67: 31-35.

[47] SU CHAO-TON, HSIAO YU-HSIANG, CHANG CHIA-CHIN. Parameter optimization design for touch panel laser cutting process. IEEE Transactions on Automation Science and Engineering, 2012, 9(2): 320-329.

[48] KIM KWANG-RYUL, KIM JAE-HOON, FARSON DAVE F, et al. Hybrid laser cutting for flat panel display glass. Japanese Journal of Applied Physics, 2008, 47(8): 6978-6981.

[49] TSAI CHWAN-HUEI, CHANG WEI-HAN. Pulsed laser breaking technique for glass substrates. Proceedings of SPIE, 2009, 7204: $1-9$.

[50] WANG XU-HUANG, YAO JIAN-HUA, ZHOU GUO-BIN, et al. Research of the technology of laser cutting LCD glass substrates based on thermal cracking method. Laser Technology, 2011, 35(4): 472-476.

[51] WANG XU-HUANG, YAO JIAN-HUA, ZHOU GUO-BIN, et al. Numerical simulation and experiment of laser cuttingLiquid crystal display glass substrates . Chinese Journal of Lasers, 2011, 38(6): 1-5. 